\title{
Machine Learning for the Dynamic Scanning Transmission Electron Microscopy Experiment on Solid State Transformations
}

\author{
Maxim Ziatdinov ${ }^{1,2}$, Artem Maksov ${ }^{1,2,3}$, Ondrej Dyck ${ }^{1,2}$, Stephen Jesse ${ }^{1,2}$, and Sergei V. Kalinin ${ }^{1,2}$ \\ 1. Center for Nanophase Materials Sciences, Oak Ridge National Laboratory, Oak Ridge, USA. \\ 2. Institute for Functional Imaging of Materials, Oak Ridge National Laboratory, Oak Ridge, USA. \\ 3. Bredesen Center for Interdisciplinary Research, University of Tennessee, Knoxville, USA.
}

Understanding elementary mechanisms behind the solid-state phase transformations and reactions is a key to developing and advancing technological applications ranging from battery and fuel cell operation to formation and operation of electro-resistive devices. Recent advances in (scanning) transmission electron microscopy ((S)TEM) allow the visualization of solid-state transformations in materials via observations of atomic motion induced by thermal or chemical stimuli or electron beam in real time. Yet despite large volumes of data generated in STEM experiments, the available to date analytical tools do not allow to learn much from the collected data. Here we developed a deep-learning-based approach for elucidating the solid-state transformations and reaction pathways from dynamic STEM data on 2dimensional Mo-doped $\mathrm{WS}_{2}$.

We adopt a deep convolutional neural network that can output pixel-wise classification maps and train it to find defects that break lattice periodicity. Specifically, we train a convolutional neural network using only the (properly augmented) $1^{\text {st }}$ frame from the dynamic STEM experiment, when macroscopic periodicity is still maintained, and each defect can be readily discovered providing the "ground truth" for network training. Thus trained network then uses its generalization abilities [1] to discover other lattice defects in the rest of the frames which may not necessarily be a part of the initial training set [2].

The trained deep learning model allows extracting $\sim 10^{3}-10^{4}$ defects from the typical dynamic STEM dataset, which can be studied further by categorizing them into different types (classes) using unsupervised learning algorithms such as $k$-means and Gaussian mixture models. We then proceed to analyzing changes in the concentration of each defect type as a function of time and trajectories of the defects, which are a key for understanding physiochemical processes in the solid-state matter under the e-beam irradiation. The defects show wide range of behaviors, including the evolving coupling between defects of different types (e.g., coupling between Mo dopants and S vacancies). In addition, because each class contains sufficiently large number of individual images of isolated defect it becomes possible to search for statistically significant distortions of an average "unit cell" structure for selected defects. We specifically demonstrate how to use the principal component analysis to search for distortions from average "unit cell" for defects associated with Mo dopant.

Finally, we discuss steps necessary for including specific theory constraints, such as transition probabilities between defect structures, explicitly into our deep-leaning-based modelling. Because the current methods treat observed lattice defects as collections of pixels, without "understanding" the physics behind the observations, we propose improving a classification accuracy is by integrating Markov model into the search and identification/classification of atomic and defect structures. The Markov model can be guided by the theoretical calculations of interaction potentials on the atomic level, enforcing physical constraints to transition probabilities of various atomic and molecular configurations and effects of electron beam on the matter. This would be an important step towards creating a fully- 
autonomous, AI microscope that is making decisions based on the knowledge of physics that it was "taught" [3].

References:

[1] M. Ziatdinov et al., ACS Nano 11 (2017), p. 12742.

[2] A. Maksov et al., to be submitted.

[3] The work was supported by the U.S. Department of Energy, Office of Science, Materials Sciences and Engineering Division (M.Z., S.V.K.). This research was conducted and partially supported (O. D., S.J.) at the Center for Nanophase Materials Sciences, which is a US DOE Office of Science User Facility.

(a)

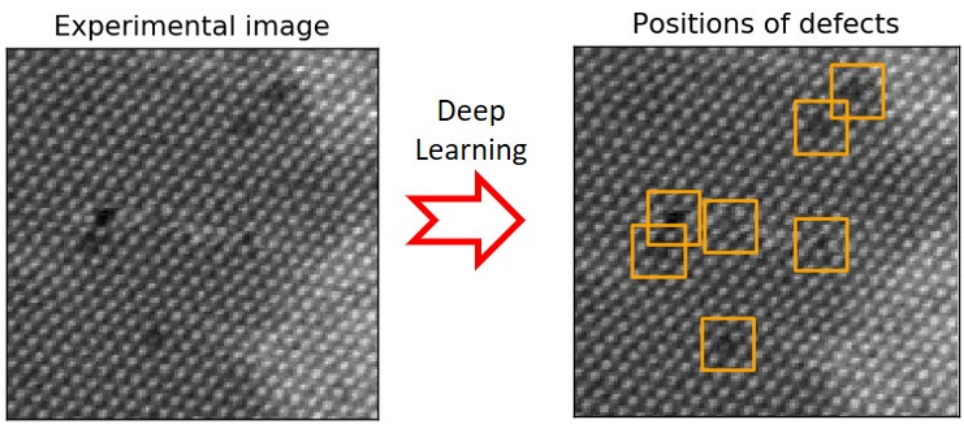

(b) Hierarchical Clustering Dendrogram (truncated)

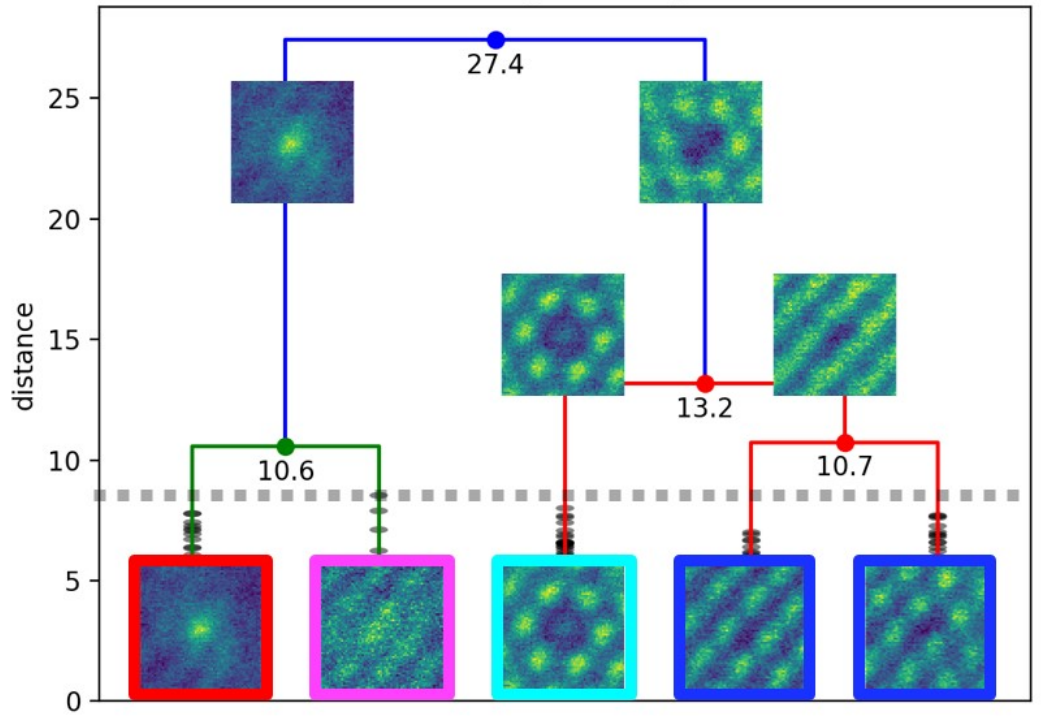

Figure 1. (a) Using a deep learning model to identify positions of defects that break lattice periodicity from STEM data on $\mathrm{WS}_{2}$. (b) Hierarchical clustering dendrogram describing different classes of defects extracted from STEM movie on $\mathrm{WS}_{2}(\sim 100$ frames $)$. 